\title{
Propuesta de una técnica de comprobación de objetivos de aprendizaje para unidades didácticas mediante el uso de software
}

\author{
Proposal for a Software-Based Learning Objective Verification Technique for Didactic Units \\ Proposta de técnica de verificação de objetivos de aprendizagem para unidade didática \\ utilizando software
}

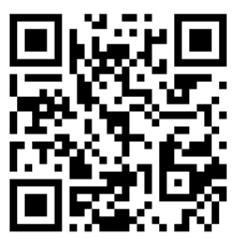

Sarita Poltronieri-Méndez Universidad Estatal a Distancia

San José, Costa Rica spoltronieri@uned.ac.cr https://orcid.org/0000-0003-3151-180X

Gabriela Garita-González Universidad Estatal a Distancia San José, Costa Rica ggarita@uned.ac.cr https://orcid.org/0000-0002-7813-2770

Recibido • Received • Recebido: 24 / 01 / 2020

Corregido • Revised • Revisado: 24 / 03 / 2021

Aceptado • Accepted • Aprovado: 07 / 04 / 2021

\begin{abstract}
Resumen
Objetivo. El estudio tiene el objeto de proponer una técnica de revisión para la comprobación de los objetivos de aprendizaje en la unidad didáctica, en contraste con la propuesta curricular de la asignatura de la Universidad Estatal a Distancia (UNED, Costa Rica). Metodología. Presenta un enfoque mixto, que hace uso de dos herramientas principales la sistematización de la experiencia por el orden en que sucedió y el grupo focal con 14 personas expertas (11 mujeres y 4 hombres) que realizaron un análisis de confiabilidad del diseño propuesto. Análisis de resultados. El principal hallazgo fue la validación de una técnica de revisión que apoya el proceso de producción de una unidad didáctica. Esta consiste en un proceso de seis pasos que emplea, entre ellos, una rúbrica de evaluación e indicadores, que permiten reflejar el resultado final en un porcentaje de comprobación del desarrollo de los objetivos de aprendizaje de una asignatura en una unidad didáctica. Conclusión. Es posible transformar un proceso, habitualmente, manual a una forma semi automatizada por la incorporación del paquete informático Microsoft ${ }^{\circledR}$ Office Excel, que facilita una comprobación parcial o total de los objetivos desarrollados en la UD y contribuye a la estandarización de procesos actuales.
\end{abstract}

Palabras claves: Educación a distancia; diseño curricular; objetivos de aprendizaje, unidad didáctica; UNED. 
http://doi.org/10.15359/ree.25-2.19

http://www.una.ac.cr/educare

educare@una.ac.cr

\begin{abstract}
:
Objective. The study aims to propose a revision technique for verifying learning objectives in didactic units (UD), in contrast to the subject's curricular proposal at the Distance State University of Costa Rica (UNED). Method. Based on a mixed approach, two main tools were used: a systematization of experiences and an expert focus group (11 women and 4 men) that was conducted to analyze the reliability and the proposed design. Analysis of results. The main finding was the validation of a revision technique that supports the didactic unit production process. This revision technique is a six-step process that involves, among others, an evaluation rubric and indicators that deliver the final result as a percentage of the verification of the course's development of its learning objectives within a didactic unit. Conclusion. Implementing the Microsoft ${ }^{\circledR}$ Office Excel software helps transform what is usually a manual process into a semi-automated form. This provides a partial or total verification of the learning objectives developed in the UD and contributes to the standardization of the current processes.
\end{abstract}

Keywords: Curricular proposal; Distance education; Didactic unit; Learning objectives; UNED.

\begin{abstract}
Resumo:
Objetivo. O objetivo do estudo é propor uma técnica de revisão para verificar os objetivos de aprendizagem na unidade didática, em contraste com a proposta curricular da disciplina da Universidade Estadual a Distância (UNED, Costa Rica). Metodologia. Apresenta uma abordagem mista, que utiliza duas ferramentas principais: a sistematização da experiência na ordem em que ocorreu e o grupo focal com 14 especialistas (11 mulheres e 4 homens) que analisaram uma análise de confiabilidade do desenho proposto. Análise dos resultados. A principal descoberta foi a validação de uma técnica de revisão que suporta o processo de produção de uma unidade didática. Isto consiste num processo de seis etapas que utiliza uma rubrica de avaliação e indicadores, que permitem refletir o resultado final numa percentagem de verificação do desenvolvimento dos objetivos de aprendizagem de uma disciplina numa unidade didática. Conclusão. É possível transformar um processo, geralmente manual, em um formulário semiautomático, transformando o recurso de software Microsoft ${ }^{\circledast}$ Office Excel, que facilita a verificação parcial ou total dos objetivos desenvolvidos na UD e contribui para a padronização dos processos atuais.
\end{abstract}

Palavras-chave: Educação a distância; Objetivos de aprendizagem; Proposta curricular; Unidade didática; UNED.

\title{
Introducción
}

La educación a distancia es una modalidad educativa, en la cual la presencialidad en un lugar de estudios no se requiere. Esta propicia el aprendizaje desde el principio del estudiantado como eje central, quien es el responsable de su propio aprendizaje. De igual forma, la institución que lo acoge debe brindarle los materiales didácticos necesarios para que dirija y autorregule el estudio de la información, actualmente, se cuenta con apoyo de la tecnología.

Uno de los materiales didácticos facilitados por la Universidad Estatal a Distancia (UNED) es la Unidad Didáctica (UD). Esta se diferencia de un libro de texto, debido a la intencionalidad del contenido y a la complejidad didáctica, ya que es producida para apoyar directamente una 
asignatura específica en un nivel académico, por tanto, integra mediación pedagógica y orienta con elementos de enseñanza-aprendizaje al estudiantado, de forma que este aprecia al tutor en el texto mientras lo estudia. No obstante, al igual que los libros de texto, posee criterios de selección de títulos, tales como la calidad de los contenidos (Giménez-Toledo et al., 2018).

En la UNED, la creación de la UD está dirigida por productores académicos del Programa de Producción de Materiales Didácticos Escritos (PROMADE) y fundamenta su desarrollo en el currículo de la asignatura que, para la UNED, es llamado Diseño Curricular (DC). Sin embargo, este proceso de creación se realiza de forma manual, con base en los criterios individuales del personal a cargo, quienes tienen un procedimiento, maduro y claro; pero este difiere de una persona productora académica a otra, es decir, no posee un lineamiento claro o específico a seguir. Es así como este proceso de revisión, basado en la experiencia, dificulta la curva de aprendizaje del nuevo personal que se incorpora al programa; también, genera confusión para la toma de decisiones, por parte de los actores que conforman el equipo de producción (personas productoras académicas, personas encargadas de cátedra; personas encargadas de programa de grado, de posgrado o de extensión; especialista en contenidos; uno o más autores o autoras).

De esta manera, se destaca la importancia de proponer técnicas de revisión y herramientas que permitan comprobar que la UD de la UNED desarrolle su contenido en función de los objetivos de aprendizaje propuestos en el DC de la asignatura, además, de contar con una mediación pedagógica suficiente y clara.

Ahora bien, de esa integración entre el DC y elaboración de la UD, surge la pregunta: ¿Cómo diseñar una técnica que apoye al personal productor académico en la comprobación de objetivos de aprendizaje en la unidad didáctica? Por ello, esta investigación propone una forma que facilite responder a esta interrogante con el uso de la tecnología. Un logro supone facilitar la labor del equipo de producción, que la persona productora académica que recién se integra tenga un proceso de adaptación progresivo y finalice la UD con mayor calidad.

\section{Marco teórico}

Esta sección se fundamentará desde tres perspectivas: la educación a distancia, el programa PROMADE y los paquetes informáticos, los cuales serán descritos a continuación.

\section{La educación a distancia}

Esta ha evolucionado lo largo degeneraciones deinnovación y detecnología. Para Garrison (1985, 1989), cada generación es caracterizada por la mediación (las actividades utilizadas y el desarrollo de los contenidos). Este tipo de modelo de educación sitúa al estudiantado como el responsable de su aprendizaje, el personal tutor es quien guía y evalúa su progreso, entre tanto, el medio de comunicación tecnológico es un recurso que facilita el aprendizaje. En este contexto, 
http://doi.org/10.15359/ree.25-2.19

http://www.una.ac.cr/educare

educare@una.ac.cr

la educación a distancia brinda una respuesta viable a la extensa demanda de educación, en especial para aquellas personas que no pueden atender a una educación presencial (Chaves Torres, 2017) y dependen del recurso didáctico para su aprendizaje.

En este orden de ideas, se hace necesario conceptualizar el recurso didáctico como la variedad de materiales elaborados para facilitar la información y para guiar el proceso de aprendizaje, la capacitación, la mejora de habilidades, la motivación y la autoevaluación. Continuando con el concepto, se denomina también como material didáctico, recurso, auxiliar didáctico, medios didácticos (Cabero, 2001).

Específicamente, la UNED señala en su Modelo pedagógico, como un elemento esencial a la unidad didáctica modular (UDM) (UNED, 2004a). La cual, según el Plan Académico 2001-2006, se define como una "estrategia curricular que considera los recursos [educativos] disponibles en la institución, los estilos y ritmos de aprendizaje ... de los estudiantes, la [universalización] de los medios empleados y la posibilidad de acceso de los estudiantes a dichos medios" (Plan Académico UNED 2001-2006 citado por UNED, 2004b, p. 4).

Uno de los elementos de esta UDM es la UD, que es un componente primordial del aprendizaje dentro del modelo pedagógico empleado. La UD se entiende como pequeños volúmenes que poseen una parte importante del curso que, por ellos mismos, son una unidad, sesión 115 de la Junta Universitaria (UNED, 1978). También, se considera el material didáctico escrito que posee y presenta los contenidos que se desarrollan en una asignatura; los cuales han sido determinados, de previo, por un grupo de especialistas durante el proceso de planificación curricular (UNED, s. f.).

Es así como la UD acompaña a estudiantes y al personal tutor durante el periodo lectivo. Entonces, la UNED dispone de recursos didácticos que promueven el aprendizaje; asimismo, el personal tutor es quien guía, evalúa su progreso y se relaciona con el estudiantado en distintos momentos del periodo lectivo (UNED, 2009). Cada UD cumple un propósito específico definido en el diseño curricular de la asignatura, el cual se conceptualiza como una estructura de planificación de un currículo (planes de estudio) que integra los procesos de selección, de organización y de evaluación de los contenidos curriculares.

Para la UNED, el DC se basa en lo expuesto por Corrales et al. (2006) y se entiende como el documento de referencia que presenta el plan de estudios en sus aspectos operacionales, ya que enfatiza en los objetivos por alcanzar en la asignatura, sus estrategias, los materiales didácticos necesarios y el tipo de evaluación que se empleará. Es así como el contenido que desarrolla la UD debe ir de la mano con lo que el DC de cada asignatura señala.

Cuando se produce una UD, se destacan elementos que permiten planificar el alcance de la producción de cada una de ellas y serán esenciales en el diseño de la técnica de comprobación de los objetivos que se detalla en esta investigación. 


\section{PROMADE}

La Dirección de Producción de Material Didáctico (DPMD) cuenta con el Programa de Producción de Material Didáctico Escrito (PROMADE), creado en 1978. Este último es asesor y líder de las producciones del material didáctico escrito que provee la UNED al estudiantado. PROMADE cumple, en la universidad, con el objetivo de crear productos escritos de calidad para las asignaturas de las carreras. Entre los principales materiales didácticos que se elaboran, están: las guías de estudio, los materiales complementarios, los manuales de laboratorio y las unidades didácticas. Para producirlos, se conforma un equipo de producción (interdisciplinario), compuesto por personas productoras académicas (equipo editor); personas encargadas de cátedra; personas encargadas de programa de grado, de posgrado o de extensión; especialista en contenidos; uno o más autores o autoras. Villalobos-Rodríguez et al. (2020) detallan las funciones de cada actor mencionado y las etapas del proceso de producción de la UD.

Las etapas o fases del proceso de producción establecidas son cuatro. Resultan de nuestro interés inmediato las primeras dos. La primera, consiste en la preescritura que involucra la elaboración del plan global, el cual es un documento que incluye la propuesta de contenidos y que elabora el autor o autora luego de analizar el diseño curricular de una asignatura; al mismo tiempo, incluye un cronograma de cumplimiento. El proceso de cocreación del plan global incluye revisiones del equipo de producción. La segunda fase se denomina el primer borrador (PB), la cual culmina con una versión preliminar de la UD. La tercera consiste en el proceso de validación de la versión preliminar, y la cuarta etapa produce la versión final, que es traslada a la editorial universitaria.

Tal como se indicó, la UD es un texto mucho más completo cuya mediación integra las explicaciones que, en clase, haría la persona tutora. Por consiguiente, la UD es flexible, en cuanto a que le permite al estudiantado dirigir su autoaprendizaje en el momento que pueda emprender la lectura, sin necesidad de apoyo presencial. Es así como la creación de una UD es compleja y tiene su base de creación en el diseño curricular mismo que cumple un objetivo para una asignatura. Entre los elementos que enmarcan el alcance de la UD, se destaca la unidad temática, pues se creó para cumplir un objetivo de aprendizaje.

Ahora bien, se define el contenido como el grupo de actitudes, destrezas y habilidades que facilitan el alcance de los objetivos y adquisición de competencias, en cada etapa de aprendizaje (Gobierno de España, Ministerio de Educación y Formación Profesional, 2018). Precisamente, el desarrollo de dicho contenido es el trabajo principal en la creación de una UD y es esta el objeto principal de estudio en la propuesta que aquí se presenta.

\section{Paquetes informáticos}

Estos han sido ampliamente usados para el aprendizaje en la educación superior, no obstante, aún no alcanzan el máximo esperado. García Sánchez et al. (2016) señalan que su avance ha propiciado el desarrollo de entornos amigables y funcionales con base en las 
http://doi.org/10.15359/ree.25-2.19

http://www.una.ac.cr/educare

educare@una.ac.cr

necesidades del público usuario, generan softwares específicos, para lo cual, primero se debe determinar el concepto o la preproducción que esquematiza lo que se espera desarrollar.

Entre tanto, los paquetes informáticos de oficina han sido comúnmente utilizados para resolver el trabajo diario, sin la necesidad de ahondar en el desarrollo de un software complejo. Por ejemplo, el uso del software de la hoja de cálculo Microsoft ${ }^{\circ}$ Office Excel, que permite organizar varias hojas que integran cálculos y fórmulas matemáticas para semiautomatizar procesos, facilita que tareas manuales sean esquematizadas y estandarizadas para servir como apoyo a las labores diarias de quienes los utilizan y, en este caso, los sujetos productores académicos.

Por lo tanto, para esta investigación, se programaron, de forma macro, algunos elementos que, con fórmulas matemáticas, permiten esquematizar los resultados y lograr una representación numérica para ser comparada con indicadores y, así, permitir el análisis de la información. El detalle de esta programación fue expuesto por Garita González y Poltronieri Méndez (2020).

\section{Metodología}

La metodología se realizó desde el enfoque mixto, en el cual el planteamiento emplea tanto aspectos cualitativos como cuantitativos, que representan un conjunto de análisis sistemáticos, empíricos y críticos de la recolección de los datos; con el propósito de generar un producto maximizado con toda la información encontrada, al igual que su integración y discusión conjunta (Hernández Sampieri y Mendoza Torres, 2018). De esta manera, se formalizó la sistematización de la experiencia que permitió impulsar el diseño de una propuesta de técnica de comprobación de objetivos de aprendizaje para las unidades didácticas generadas en el programa PROMADE de la UNED. Para esto, es necesario comprender que la propuesta se alimenta de dos elementos principales para el proceso de producción de unidades didácticas, a saber: el diseño curricular y el plan global. Estos elementos en conjunto delimitan el contenido y los objetivos de la obra por desarrollar.

Específicamente para la labor que realiza PROMADE, se parte del hecho de que el contenido del diseño curricular responde, de forma directa, al cumplimiento de los objetivos de aprendizaje que él indica. Razón por la cual el objetivo, el tema, su importancia y profundidad van de la mano.

Con base en los principios mencionados, el proceso de esta técnica de comprobación se basó en los criterios de calidad mencionados en la misión y visión de PROMADE. En ellos descansa la estandarización y calidad, basadas en las características de mediación pedagógica, pertinencia y contenidos; las cuales están, estrictamente, relacionadas su efectividad. Se delimita su alcance en la comprobación del cumplimiento de los objetivos de aprendizaje de las UD (Garita González y Poltronieri Méndez, 2020).

6 
El proceso de sistematización inició en enero de 2017 con el desarrollo de tres etapas. La primera es el objeto de conocimiento, para este contexto, la unidad didáctica. Se empleó un documento semiautonatizado de Microsoft ${ }^{\circ}$ Office Excel para apoyar la revisión manual del proceso de producción de la UD (Garita González y Poltronieri Méndez, 2020). La segunda son actores directos e indirectos, de forma directa, formado por las personas productoras académicas (personal editor) y de forma indirecta, por las personas encargada de cátedra, persona encargada de carrera, personas autora y especialista. La tercera es la situación inicial, donde, el proceso de producción se realiza totalmente manual. Tal como se mencionó, es un procedimiento consolidado y claro para el personal experimentado; sin embargo, se realizan acciones basadas en criterios individuales de forma que discrepan entre personas productoras académicas. Esto condujo a las investigadoras a diseñar una técnica, con el fin de comprobar objetivos de aprendizaje y poder nivelar el conocimiento de años y experiencia.

Lo dicho hasta aquí resume que la técnica propuesta cuenta con intencionalidad, ya que facilita la comprobación de los objetivos de aprendizaje en la UD en contraste con el diseño curricular que son dos elementos protagonistas. También, busca una situación final, es decir, puede utilizarse durante todo el proceso de producción que se desarrolla en un promedio de dos años. Además, facilita la reflexión individual y grupal del equipo de producción, al permitir la comprobación de objetivos en la UD de forma visual y clara. Refleja el avance de la producción y del estado de los contenidos por mejorar hasta el término del trabajo con la entrega a la editorial universitaria EUNED.

\section{Análisis de confiabilidad}

Se efectuó por el método de juicio experto. Por medio de tres talleres, se contó con la partición voluntaria de 14 personas productoras académicas (personal editor) (11 mujeres y 4 hombres), se eligieron como muestra intencional por dos características principales. La primera, porque son personas académicas con amplia experiencia en la educación a distancia y de creación de materiales didácticos y, la segunda, porque que promedian de 12 a 14 años de experiencia en sus funciones. En el grupo focal se demostró la técnica propuesta y se permitió realizar ensayos con ella. El proceso de revisión se sometió a estudio desde dos criterios de calidad: la validez del diseño y la objetividad de la técnica.

\section{Objetivo general}

Proponer una técnica de revisión para la comprobación de los objetivos de aprendizaje en la unidad didáctica, en contraste con la propuesta curricular de la asignatura de la UNED (Costa Rica). 
http://doi.org/10.15359/ree.25-2.19

http://www.una.ac.cr/educare

educare@una.ac.cr

\section{Resultados análisis y discusión}

Esta sección se divide en nueve puntos. Los primeros tres contextualizan los fundamentos por emplear para la comprobación de los objetivos. Los restantes seis muestran la aplicación de la técnica.

\section{Criterios para la creación de la UD}

Para esta pesquisa, se consideraron los criterios para la creación de la UD, de acuerdo con su definición y el avance en el uso de sistemas digitales actuales que facilitan el empleo de ilustraciones. Esta información se integró a los criterios de la propuesta del Reglamento del sistema de producción de obras de la Universidad Estatal a Distancia (UNED, Programa de Producción de Material Didáctico Escrito [PROMADE] s. f.). El detalle que más nos ocupa en esta investigación se relaciona con la extensión de la UD, a saber:

i. Un máximo de 50000 palabras, para obras con hasta $30 \%$ de ilustraciones dentro de la caja de texto.

ii. Un máximo de 70000 palabras para obras con un porcentaje inferior al $5 \%$ de las ilustraciones, o ilustraciones que solo van en columna auxiliar.

Lo anterior equivale a una copia o ejemplar de 150 a 200 páginas publicadas, en formato 8 $\times 10$ pulgadas, con cajas de texto de 400 palabras por página y de estando ya consideradas las páginas blancas, los retiros, etc. (p. 31)

\section{Plan global}

Este se compone de dos partes, la propuesta de contenidos de la UD y un cronograma de trabajo. El primero se fundamente en el DC de la asignatura; y el segundo, en la fecha final para la cual será empleado el material (Guerra González, 2016). En él se incluyen únicamente los temas que el equipo de producción acuerda que serán desarrollados en la obra.

\section{Escala de valoración del diseño curricular}

El diseño curricular es la guía y delimita el alcance de la UD. La escala de valoración puede tener múltiples nombres; no obstante, esta hace referencia a un proceso de escalamiento en el cual una persona brinda una categoría de un conjunto de estas, ya sea de frecuencia o de cantidad, a un estímulo (Matas, 2018).

Se destaca, en este apartado, la distribución de tiempo en horas con base en una asignación de puntos en el DC y sobresalen dos elementos que caracterizan la temática que se aborda en la UD, a saber: la importancia y la profundidad. En el Tabla 1, se detalla un ejemplo, en el cual se observa, en gris claro, sus criterios y; en gris oscuro, se resaltan las dos columnas que serán empleadas. 
http://doi.org/10.15359/ree.25-2.19

Tabla 1: Distribución de horas para una asignatura de la UNED que integra el diseño curricular

\begin{tabular}{ccccccc}
\hline Tema & Importancia & $\begin{array}{c}\text { Complejidad de } \\
\text { las actividades }\end{array}$ & Profundidad & $\begin{array}{c}\text { Total de } \\
\text { puntos }\end{array}$ & $\begin{array}{c}\text { Porcentaje } \\
\text { tema }\end{array}$ & $\begin{array}{c}\text { Tiempo } \\
\text { horas }\end{array}$ \\
\hline Tema I & 3 & 2 & 2 & 7 & 14,9 & 20,1 \\
Tema II & 3 & 3 & 3 & 9 & 19,2 & 26,0 \\
Tema III & 4 & 4 & 4 & 12 & 25,5 & 34,4 \\
Tema IV & 4 & 4 & 4 & 12 & 25,5 & 34,4 \\
Tema V & 3 & 2 & 2 & 7 & 14,9 & 20,1 \\
\hline & & TOTAL & & 47 & 100 & 135 \\
\hline
\end{tabular}

Un crédito equivale a 45 horas de trabajo en educación a distancia (CONARE).

$\%$ tema $=$ total de puntos por tema/totales de puntos * 100 . Tiempo (horas) $=45 \mathrm{~h} *$ (\% tema) $/ 100$.

Criterios en puntos: $1=$ poca, $2=$ media, $3=$ alta, $4=$ suprema

Nota: Adaptado de Guía para el diseño y rediseño curricular de asignaturas (UNED, Programa de apoyo curricular y evaluación de los aprendizajes [PACE], 2018).

La Tabla 1 detalla los cinco temas por desarrollar durante la asignatura y sus criterios. En este caso, el tercer y cuarto tema son de importancia, complejidad y profundidad suprema para la asignatura, en relación con los temas primero, segundo y quinto; por tanto, tienen el mayor tiempo asignado.

Específicamente, cada criterio se explica de la siguiente forma: Importancia del tema, que concierne a la relevancia del tema con respecto al resto de las temáticas de la asignatura y su relación con la formación profesional o área disciplinar. Complejidad de las actividades, la cual se establece según las experiencias de evaluación y el tiempo promedio que se estime, así el estudiantado invertirá tiempo en trabajo académico para su ejecución. Profundidad temática, que depende de la amplitud del alcance del objetivo de aprendizaje, el cual se enuncia en el verbo (UNED, PACE, 2018).

Una vez clara la fundamentación, con base en la Tabla 1, y con las columnas 2 (importancia del tema) y 4 (profundidad del tema) de la Tabla 1, se calcula la extensión del contenido de cada tema para la UD. Es importante aclarar, que no se considera la columna 3 (complejidad de las actividades) de la Tabla 1, debido a que, la UD no es un instrumento de evaluación, por el contrario, es un material didáctico que facilita el aprendizaje al estudiantado. El cálculo a partir del ejemplo utilizado se expone en la Tabla 2. 
http://doi.org/10.15359/ree.25-2.19

http://www.una.ac.cr/educare

educare@una.ac.cr

Tabla 2: Distribución de palabras máximas por tema, de una unidad didáctica de la UNED. Se muestran dos posibles opciones

\begin{tabular}{|c|c|c|c|c|c|c|}
\hline \multirow[t]{2}{*}{ Tema } & \multicolumn{2}{|c|}{ Criterios } & \multirow{2}{*}{$\begin{array}{l}\text { Total de } \\
\text { puntos }\end{array}$} & \multirow{2}{*}{$\begin{array}{c}\text { Porcentaje } \\
\text { del tema }\end{array}$} & \multirow{2}{*}{$\begin{array}{c}\text { Opción } 1 \\
\text { (50 000 palabras y } 30 \% \\
\text { ilustraciones) }\end{array}$} & \multirow{2}{*}{$\begin{array}{c}\text { Opción } 2 \\
\text { (70 } 000 \text { palaras y 5\% } \\
\text { ilustraciones) }\end{array}$} \\
\hline & $\begin{array}{l}\text { Importancia } \\
\text { del tema }\end{array}$ & $\begin{array}{c}\text { Profundidad } \\
\text { del tema }\end{array}$ & & & & \\
\hline Tema I & 3 & 2 & 5 & 15,63 & 7812,5 & 10937,5 \\
\hline Tema II & 3 & 3 & 6 & 18,75 & 9375 & 13125 \\
\hline Tema III & 4 & 4 & 8 & 25,00 & 12500 & 17500 \\
\hline Tema IV & 4 & 4 & 8 & 25,00 & 12500 & 17500 \\
\hline \multirow[t]{2}{*}{ Tema V } & 3 & 2 & 5 & 15,63 & 7812,5 & 10937,5 \\
\hline & Total & & 32 & 100 & 50000 & 70000 \\
\hline
\end{tabular}

Nota: Elaboración propia.

De acuerdo con la información de la Tabla 2, se recomienda el máximo de extensión en palabras para cada tema, que debe poseer la UD de la asignatura en producción. Esto pretende proyectar la extensión de cada tema, en función del desarrollo de su objetivo en la propuesta expuesta en el diseño curricular, de forma que cada estudiante cuente con materiales realizados a la medida para la asignatura que cursa.

En la columna 6 (opción 1), se expone la distribución de palabras en caso de que el texto contenga $30 \%$ de ilustraciones; $y$, en la columna 7 (opción 2), si posee $5 \%$ de ilustraciones. Lo anterior se establece en la Ecuación 1:

Cantidad de palabras $=\frac{\left(\% \text { tema }{ }^{*} \mathrm{~N} .{ }^{\circ} \text { palabras }\right)}{100}$

Donde:

$\%$ tema $=$ porcentaje del tema

N. ${ }^{\circ}$ palabras $=$ cantidad de palabras elegidas para el tema (50 000 o 70000 )

\section{Procedimiento general}

El procedimiento general es una instrucción que brinda indicaciones, paso a paso, a través de un libro de Microsoft ${ }^{\circ}$ Office Excel con hojas programadas, para el logro efectivo de la comprobación parcial o total de la UD en versión preliminar. La información se encuentra esquematizada en la Figura 1. 
Figura 1: Procedimiento general

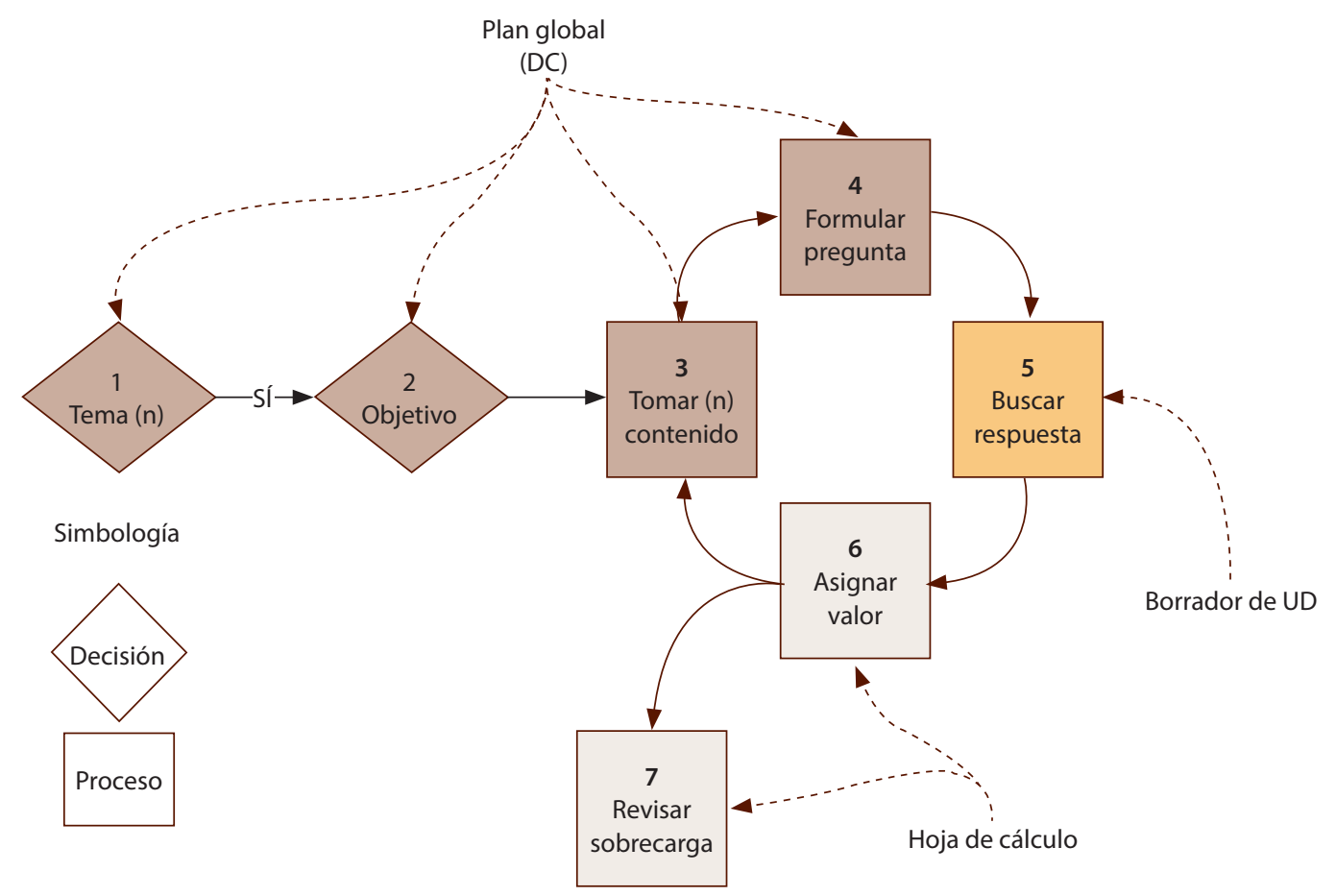

Nota: Se refleja el proceso cíclico para la comprobación de objetivos de aprendizaje, de un primer borrador de una unidad didáctica. Elaboración propia.

El procedimiento se ejecuta de la siguiente forma: se localiza un tema, se ubica su objetivo de aprendizaje, se toma un contenido de la columna Descripción general de los contenidos (DC), este contenido se transforma en pregunta (paso 3) con base en el objetivo que lo engloba y se agrega a la columna en la hoja de cálculo, se sitúa su respuesta en el PB, se analiza el texto y se asigna un valor en la hoja de cálculo y, finalmente, se verifica la sobrecarga del tema.

Este procedimiento pretende organizar el trabajo que se realiza, de forma que cualquier miembro del equipo de producción comprenda el estado en el cual se encuentra. Además, busca facilitar la lectura y la toma de decisiones con respecto al desarrollo del contenido que esta debe contener para cumplir con los objetivos propuestos para la asignatura.

\section{Formulación de pregunta (pasos del 1 al 4)}

El proceso se demuestra en la Figura 2 (con las flechas). Es importante porque cada pregunta delimita el alcance y la profundidad de la temática. Se toma como referencia el tema I, objetivo I (columna 3) y contenido 1 (columna 4). A este último se le extrae un enunciado que se transforma en pregunta (columna 5), la cual se formula con base en el nivel de complejidad según objetivo de aprendizaje (columna 3) expuesto en el DC. La pregunta formulada debe agregarse a la hoja de cálculo, en el espacio señalado para ella. 
http://doi.org/10.15359/ree.25-2.19

http://www.una.ac.cr/educare

educare@una.ac.cr

Figura 2: Plantilla de trabajo

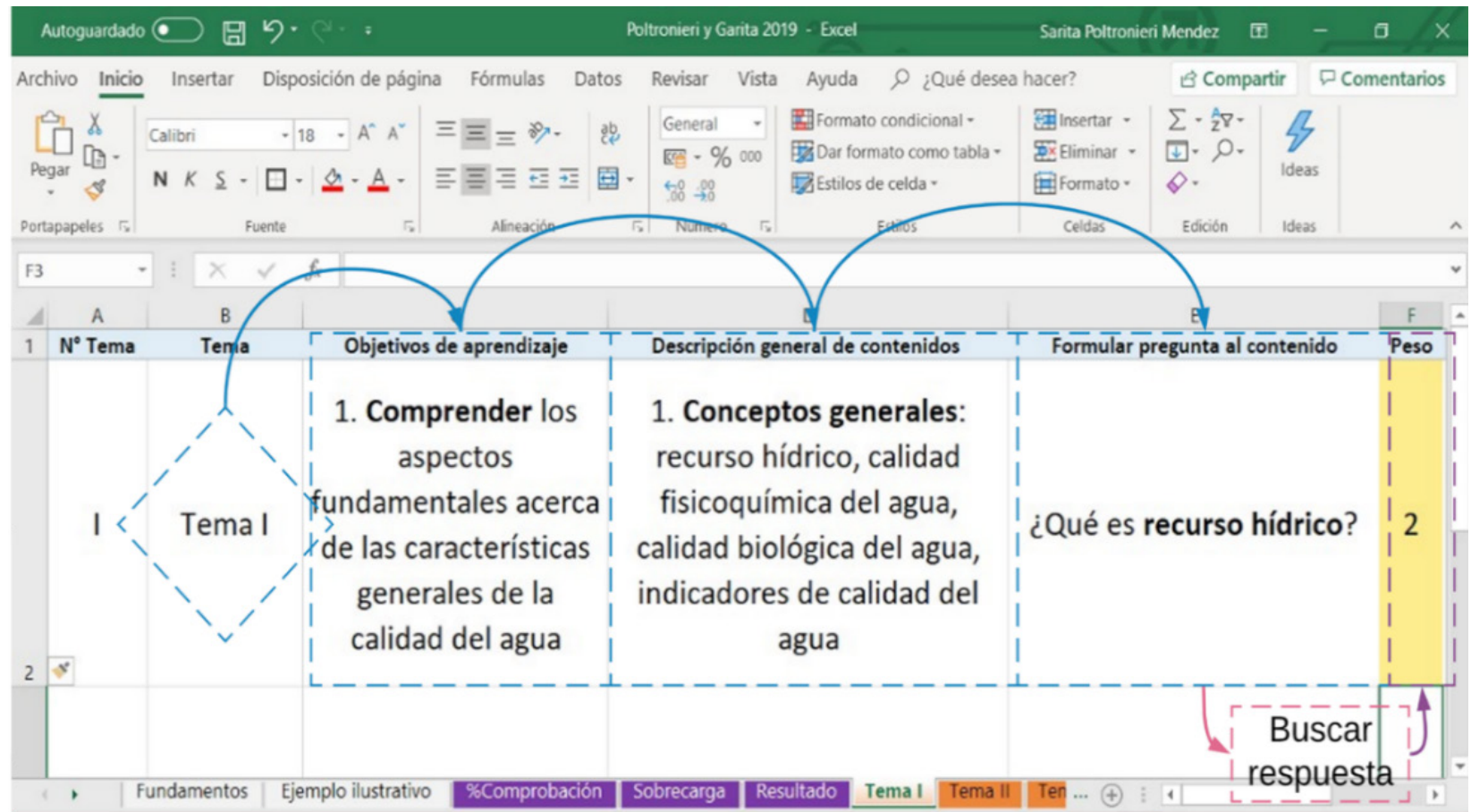

Nota: Ejemplo ficticio de procedimiento utilizado para la correspondencia entre el diseño curricular y el primer contenido del tema I de una asignatura. Elaboración propia.

De esta manera es posible denotar cuáles temas deben poseer un mayor énfasis en el desarrollo de su contenido. También, permite al equipo de producción proyectar el uso de otros recursos didácticos, para enfatizar en aquellos temas que considere más prácticos, tales como simuladores, recursos multimediales, entre otros.

\section{Rúbrica (pasos 5 y 6)}

Específicamente, una rúbrica de evaluación consiste en una matriz de valoración que contiene criterios de ejecución de una tarea y una escala que se explica textualmente. En cada casilla, se expone qué escala corresponde a cada tipo de ejecución (Cano, 2015). Para este caso en específico, consiste en asignar un valor a la pregunta formulada, a fin de brindarle respuesta luego de la lectura del PB (Figura 3, columna 6). Para esto, se empleó el método de evaluaciones sumatorias, modificado en este caso, en el cual el valor se le asigna a cada pregunta por parte de la percepción del productor académico con apoyo en su criterio experto y experiencia en la edición de textos académicos. La escala posee cuatro valores, a saber (Tabla 3): 
http://doi.org/10.15359/ree.25-2.19

Tabla 3: Escala de valoración propuesta y sujeta a validación

\begin{tabular}{|c|c|c|c|}
\hline Valor & Pregunta formulada & Contenido & \multicolumn{1}{c|}{ Mediación pedagógica } \\
\hline 3 & Sí se responde & Es suficiente, es claro & Sí posee, es suficiente \\
\hline 2 & Sí se responde & No es suficiente, no es claro & Es insuficiente \\
\hline 1 & No se responde & No se evidencia o hay plagio & No se evidencia o hay plagio \\
\hline \multirow{2}{*}{ No aplica } & $\begin{array}{l}\text { Revela que, de acuerdo con el Plan Global, el contenido temático no será abordado en la unidad } \\
\text { didáctica y no se asigna valor. }\end{array}$ \\
\hline
\end{tabular}

Nota: Elaboración propia.

Al continuar con el ejercicio de la Figura 2, el PB cumple parcialmente con las expectativas del ente productor académico. Por lo tanto, se asigna un valor 2.

La rúbrica de evaluación (valor $=3,2,1$ ) se asigna por cada pregunta generada hasta abarcar la totalidad de los contenidos establecidos en el plan global para la UD preliminar (Figura 3 ).

Figura 3: Formulación de preguntas

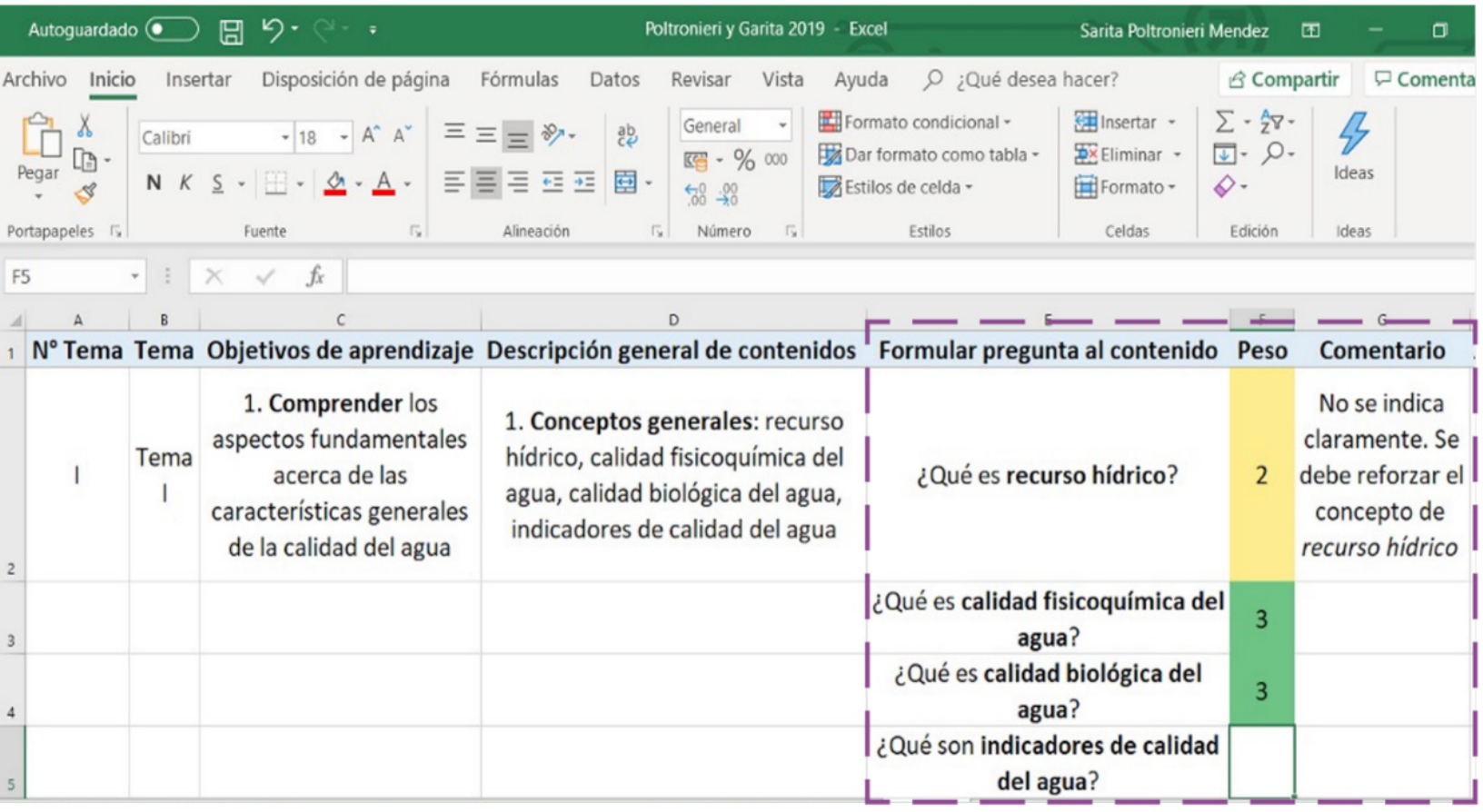

Nota: Ejemplo ficticio del proceso de formulación de preguntas y asignación de valores o peso a cada una de ellas, luego de leer el PB. Elaboración propia. 
http://doi.org/10.15359/ree.25-2.19

http://www.una.ac.cr/educare

educare@una.ac.cr

Con el propósito de visualizar el resultado de la comprobación de objetivos, se presenta como un porcentaje. La comprobación global de la unidad didáctica preliminar será llamada: porcentaje de comprobación. Esta se calcula de modo automático en la hoja de cálculo y, matemáticamente, se representa en las ecuaciones 2 y 3 :

Comprobación por tema:

$$
\% \mathrm{t}=\frac{(\Sigma P) * 100}{l * 3}
$$

Donde:

$\% \mathrm{t}=$ porcentaje de comprobación por tema $(\%)$

$\mathrm{P}=$ sumatoria de los valores asignados

I = cantidad total de contenidos por tema

Comprobación de la unidad didáctica:

$$
\%=\frac{\Sigma \% t}{N}
$$

Donde:

$\%$ = porcentaje de comprobación

$\% \mathrm{t}=$ porcentaje de comprobación por tema (\%)

$\mathrm{N}=$ cantidad total de temas del diseño curricular

Esto permite visualizar los vacíos que se encuentran en el PB del documento y realizar los ajustes necesarios para que este responda a lo requerido para la asignatura. Es decir, funciona como un método de identificación temprana de ausencia de contenidos para el cumplimiento del objetivo, al igual que permite observan si algún tema se extralimita en el abordaje propuesto en el DC.

\section{Extensión del contenido o sobrecarga (paso 7)}

La sobrecarga de información sucede cuando la cantidad de datos que una persona debe analizar para tomar una decisión supera su capacidad de procesar dicha información. Esto puede generar actitudes como ansiedad, procrastinación y evasión de información que puede ser potencialmente innecesaria desde su punto de vista (Fuertes et al, 2019).

Para efectos de esta técnica, la extensión de contenido estima la sobrecarga del texto. Motivo por el cual se considerar la extensión de los contenidos, como equilibrio en la carga académica por asignatura del estudiantado. Se debe precisar que la UD es un componente, entre otros del universo de instrumentos de aprendizaje de cada asignatura. 
http://doi.org/10.15359/ree.25-2.19

Dicho lo anterior, se considera la regla de que no debe sobrepasar la cantidad máxima de palabras establecidas para el tema (Tabla 2). Esto se genera con cada tema y se basa en una resta simple; el cálculo se refleja de la siguiente ecuación 4:

\section{Estimación de sobrecarga = opción - N. ${ }^{\circ}$ palabras}

\section{Donde:}

Opción = se refiere a la cantidad de palabras máximas por capítulo seleccionada según las dos opciones disponibles (Tabla 2).

N. ${ }^{\circ}$ palabras $=$ número de palabras por tema entregado en el PB.

En caso de tener un resultado negativo (Figura 4), el autor o autora debe analizar nuevamente el documento de borrador entregado luego de recibir la retroalimentación del equipo de producción. Este último, con base en su criterio experto, recomienda qué partes del borrador se deben sintetizar o eliminar.

Figura 4: Sobrecarga

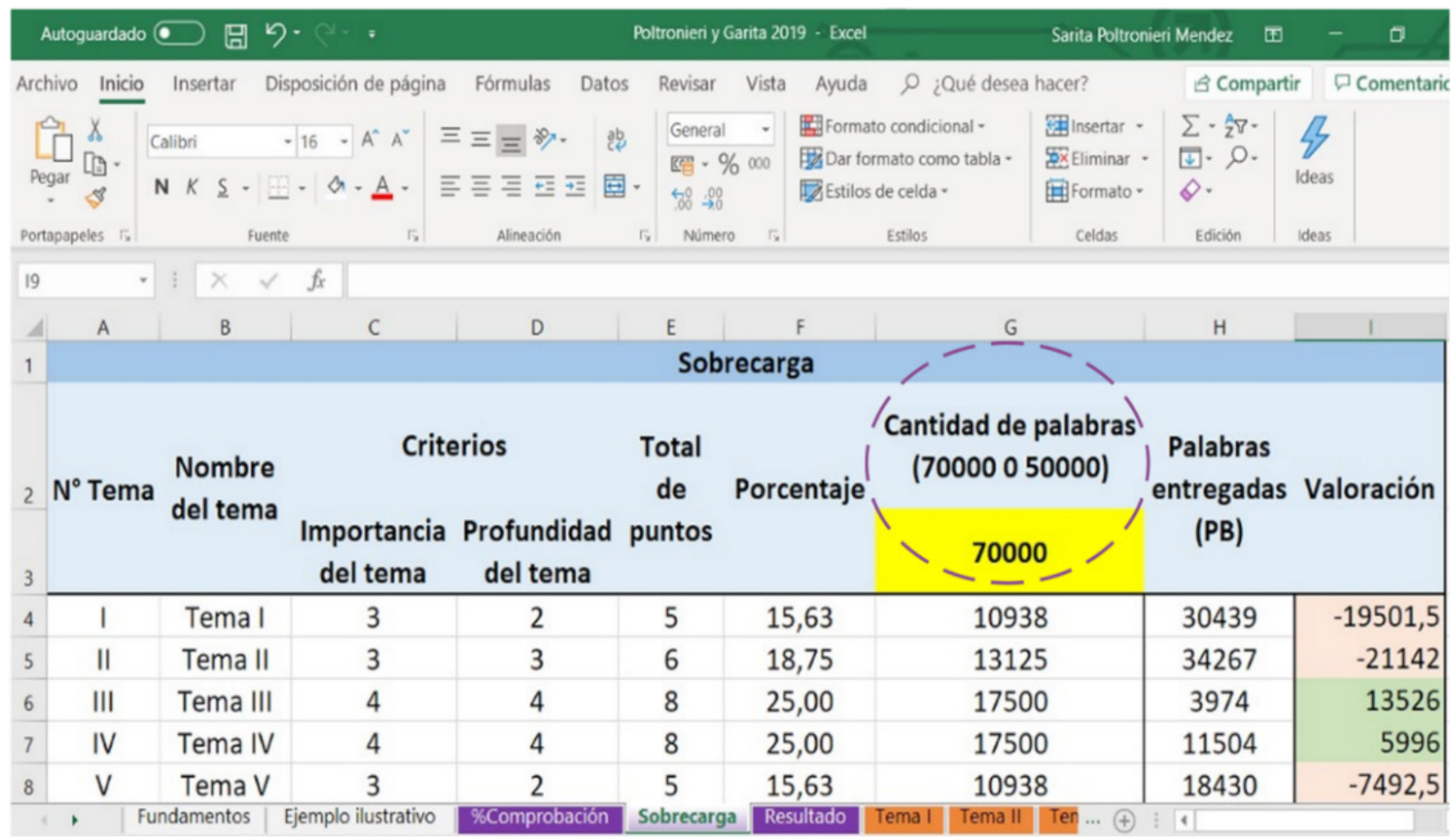

Nota: Ejemplo ficticio del proceso de la asignación del número total de palabras, proyectado para la UD y la cantidad de palabras aproximadas para cada tema. Elaboración propia. 
http://doi.org/10.15359/ree.25-2.19

http://www.una.ac.cr/educare

educare@una.ac.cr

Es necesario resaltar que los criterios establecidos por el programa para determinar la cantidad de palabras de una UD aún esta sujeta a una nueva valoración, a la luz del avance de las tecnologías de la información y comunicación (TIC) y sus aportes a la educación en la actualidad.

La importancia de contemplar la sobrecarga se vincula con la forma del pensamiento humano, desde la visión de teoría de la carga cognitiva expuesta por Sweller (2003). Esta señala que el pensamiento está fundamentado por dos tipos de información: conocimiento biológico primario y secundario; donde el último indicado considera el conocimiento asociado a la instrucción; por lo tanto, es adquirido, organizado y procesado de forma evolutiva y natural por el ser humano (Sweller, 2011).

Sweller (2011) considera la lectura y la escritura como conocimientos biológicos secundarios, por lo que se pueden utilizar para analizar algunas características del conocimiento humano, entre ellos, la susceptibilidad a la sobrecarga. También menciona que la capacidad de procesar información por parte de un individuo es fija e inalterable; además, se encuentra vinculada con los diferentes niveles de experiencia que posee. Por lo tanto, sobrepasar las capacidades del estudiantado puede afectar sus procesos de aprendizaje y desatar conductas como las mencionadas por Fuertes et al. (2019).

\section{Indicadores de comprobación}

Un indicador, precisamente, indica una condición que facilita su mejora continua, en este caso, de un texto (Leydesdorff et al., 2016). Al finalizar el ejercicio, la hoja de cálculo genera un porcentaje que representa con colores la comprobación global de la UD versión preliminar.

En esta investigación, se propone una escala de colores con indicadores de comprobación que se detalla en la Tabla 4. Por ejemplo, el color azul indica que el texto cumple más del 95\% de los objetivos de aprendizaje. De esta manera se continúa con porcentajes distribuidos en rangos de $0 \%$ a 100\%, según el grado de cumplimiento.

En la Tabla 4, se indica la propuesta para simplificar el significado de los resultados; con esto, se permite reflexionar los valores y someter los documentos a una retroalimentación positiva.

Tabla 4: Indicadores de comprobación propuestos y sujetos a mejora continua

\begin{tabular}{|l|c|c|}
\hline Indicador & Porcentaje de comprobación & Estado de la comprobación \\
\hline Azul & 95 a 100 & Muy alta \\
\hline Verde & 85 a 94 & Alta \\
\hline Amarillo & 70 a 84 & Media \\
\hline Anaranjado & 50 a 69 & Baja \\
\hline Rojo & Menos de 50 & Extremadamente baja \\
\hline Condiciones & $\begin{array}{l}\text { Cualquier indicio de copia textual sin el debido formato, plagio o autoplagio } \\
\text { condiciona el documento al indicador rojo. }\end{array}$ \\
\hline
\end{tabular}

Nota: Elaboración propia. 
http://doi.org/10.15359/ree.25-2.19

\section{Resultado}

El resultado brinda la comprobación final del PB generado de la UD con base en los indicadores de comprobación, los cuales se relacionan con el porcentaje de comprobación, según se indicó en la sección anterior. Esta información se presenta, de forma independiente, para facilitar la toma de decisiones del personal productor académico. En la Figura 5, se aprecia un ejemplo en el cual, a pesar de que el tema cuenta con un porcentaje de cumplimiento superior al $85 \%$, posee una sobrecarga importante; por lo tanto, las acciones brindadas indican la mejora de la extensión del documento. Ante esto, el documento de primer borrador de UD se le regresa a la autora o autor con la retroalimentación correspondiente que le ayude a mejorar el texto sometido a comprobación.

Figura 5: Resultado de evaluación

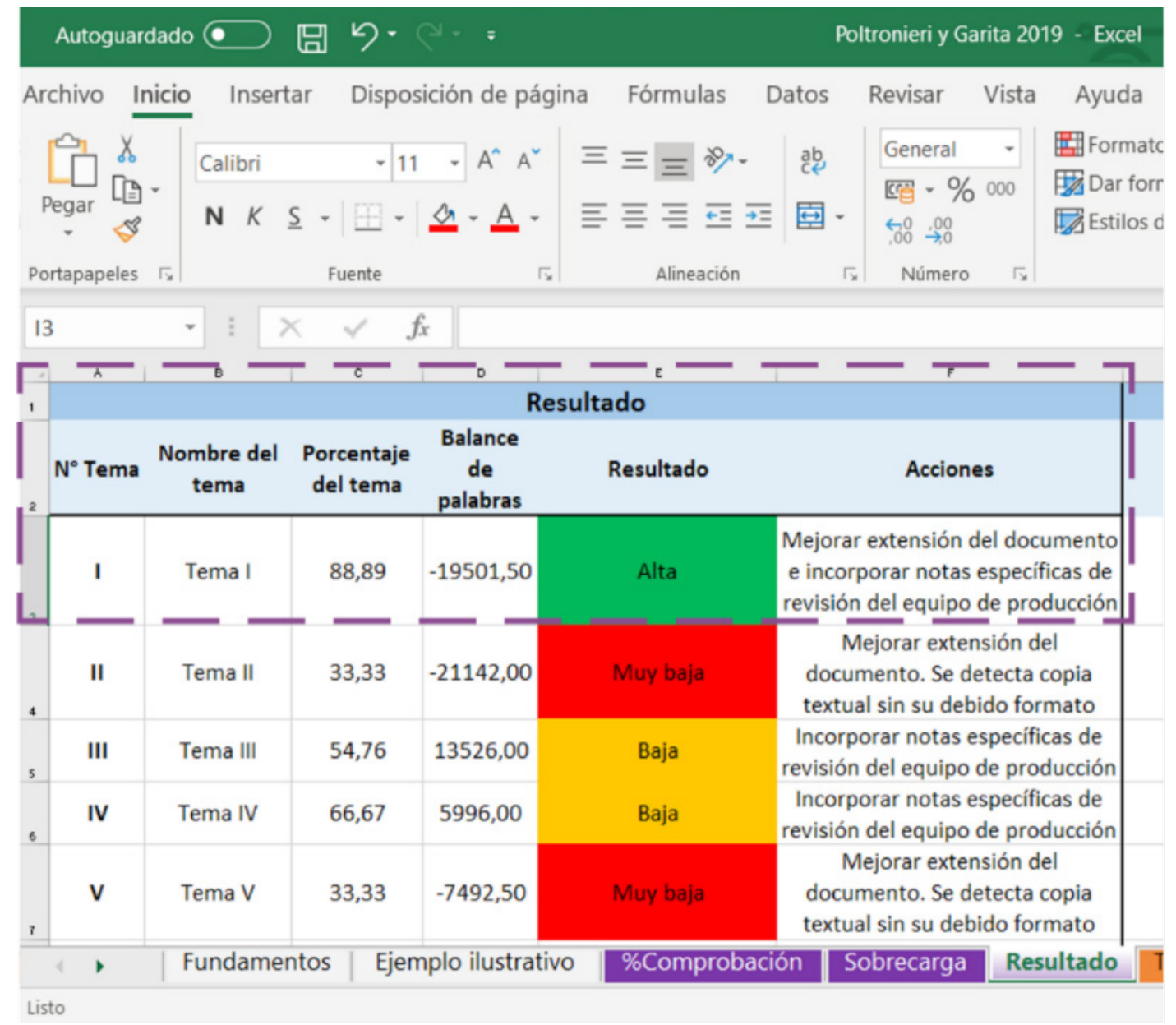

Nota: Se resalta un ejemplo de resultado de evaluación de un PB del tema I de una asignatura. Elaboración propia. 
http://doi.org/10.15359/ree.25-2.19

http://www.una.ac.cr/educare

educare@una.ac.cr

Tanto el indicador de aprobación como el resultado permiten planificar metas por alcanzar por el equipo de producción y por PROMADE en general, al tener una forma de establecer los requerimientos de cumplimiento necesarios para que una UD esté acorde con las necesidades de la asignatura. También, permite mantener un registro claro de la información para las futuras actualizaciones de cada nueva edición que se realice de la UD, e incluso, establecer metas anuales con base en indicadores claros.

\section{Conclusiones}

De acuerdo con la pregunta inicialmente propuesta, se considera posible diseñar una técnica que apoye al personal productor académico en la comprobación de objetivos de aprendizaje en la unidad didáctica. A lo largo de este documento se propuso una técnica de revisión semi automatizada con el uso del paquete informático Microsoft ${ }^{\circ}$ Office Excel, que facilita una comprobación parcial o total de los objetivos desarrollados en una UD. Una practica realizada hasta ahora de forma manual por el equipo productor académico.

La técnica favorece la revisión objetiva de una UD basada en un método de trabajo válido. Se puede decir que contribuye a la estandarización de procesos actuales del programa, que se encuentra en constante revisión y mejora, basado en su misión y visión. Específicamente, apoya la calidad del contenido de las UD, lo que contribuye con el equipo de trabajo en el proceso de producción, al estudiantado y al cuerpo docente.

Esto hace suponer que la técnica de revisión propuesta cumple con el objetivo de comprobar el abordaje de los objetivos indicados en un DC, por medio de indicadores que faciliten la mejora continua. No obstante, es recomendable someter a validaciones periódicas los componentes de la técnica, de forma que se ajuste a la actualidad del proceso de producción que se realiza en la universidad.

Es común escuchar al estudiantado universitario expresar que se sienten sobre cargados de lecturas e información en sus clases; ante esto, nace la duda de saber si, ciertamente, es demasiada como para que disfruten la lectura mientras desarrollan su aprendizaje, es decir: ¿cuánto es mucho? Por lo tanto, a futuro, las autoras recomiendan utilizar métodos más detallados para definir la capacidad lectora del estudiantado, a quien se encuentra dirigido el material que se produce. De esta manera, será posible determinar y ajustar la extensión requerida en los materiales, atendiendo sus diferentes momentos y niveles.

\section{Referencias}

Cabero, J. (2001). Tecnología educativa. Diseño y utilización de medios en la enseñanza. Paidós.

Cano, E. (2015). Las rúbricas como instrumento de evaluación de competencias en educación superior: ¿Uso o abuso? Profesorado. Revista de currículum y formación del profesorado, 19(2), 265-280. https://bit.ly/3dhBvsv 
Chaves Torres, A. (2017). La educación a distancia como respuesta a las necesidades educativas del siglo XXI. Revista Academia \& Virtualidad, 10(1), 23-41. https://doi.org/10.18359/ravi.2241

Corrales, M., Delgado, E., Umaña, A., Umaña, R. y Zamora, J. (2006). Principios teóricos y

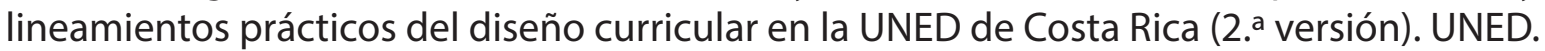

Fuertes, M. C. M., Jose, B. M. D., Nem Singh, M. A. A., Rubio, P. E. P. y de Guzman, A. B. (2019). The moderating effectsofinformationoverloadandacademicprocrastination on theinformation avoidance behavior among Filipino undergraduate thesis writers. Journal of Librarianship and Information Science, 52(3), 694-712. https://doi.org/10.1177/0961000619871608

García Sánchez, E., Vite Chávez, O., Navarrete Sánchez, M. Á., García Sánchez, M. Á. y Torres Cosío, V. (2016). Metodología para el desarrollo de software multimedia educativo MEDESME. CPU-e. Revista de Investigación Educativa, 23, 217-226. http://ref.scielo.org/mkrb3g

Garita González, G. y Poltronieri Méndez, S. (2020). TIC: Vehículo para la comprobación de los objetivos de aprendizaje en unidades didácticas para la educación superior. En XXII Congreso Internacional. Tecnología e innovación para la diversidad y calidad de los aprendizajes. EDUTEC 2019. Libro de ponencias (pp. 1674-1686. Pontificia Universidad Católica del Perú. https://bit.ly/2TVAmPU

Garrison, D. R. (1985). Three generations of technological innovation in distance education. Distance Education, 6(2), 235-241. https://doi.org/10.1080/0158791850060208

Garrison, D. R. (1989). Understanging distance education: A framework for the future. Routledge.

Giménez-Toledo, E., Tejada-Artigas, C. y Mañana-Rodríguez, J. (2018). Las editoriales universitarias iberoamericanas:Una aproximacióna su perfilya sus procesos de selección de originales. Revista Española de Documentación Científica, 41(2), 1-16. https://doi.org/10.3989/redc.2018.2.1459

Gobierno de España, Ministerio de Educación y Formación Profesional. (2018). Información general sobre la educación primaria. http://www.educacionyfp.gob.es/va/contenidos/ estudiantes/educacion-primaria/informacion-general/organizacion.html

Guerra González, J. T. (2016). La producción editorial didáctica de la Universidad Estatal a Distancia de Costa Rica: Un diagnóstico a partir del modelo de industrias de contenidos. Investigación bibliotecológica, 30(68), 125-153. https://doi.org/10.1016/j.ibbai.2016.02.007

Hernández-Sampieri, R. y Mendoza Torres, C. P. (2018). Metodología de la investigación: Las rutas cuantitativa, cualitativa y mixta. McGraw-Hill Interamericana Editores.

Leydesdorff, L., Wouters, P. y Bornmann, L. (2016). Professional and citizen bibliometrics: Complementarities and ambivalences in the development and use of indicators-a state-ofthe-art report. Scientometrics, 109(3), 2129-2150. https://doi.org/10.1007/s11192-016-2150-8 
http://doi.org/10.15359/ree.25-2.19

http://www.una.ac.cr/educare

educare@una.ac.cr

Matas, A. (2018). Diseño del formato de escalas tipo Likert: Un estado de la cuestión. Revista Electrónica de Investigación Educativa, 20(1), 38-47. https://doi.org/10.24320/ redie.2018.20.1.1347

Sweller, J. (2003). Evolution of human cognitive architecture. En B. H. Ross (Ed.), The psychology of learning and motivation (Vol. 43, pp. 215-266). Academic Press.

Sweller, J. (2011). Cognitive load theory. En J. P. Mestre y B. H. Ross (Ed.), The psychology of learning and motivation cognition in education (Vol. 55, pp. 37-76). Academic Press. https:// doi.org/10.1016/B978-0-12-387691-1.00002-8

Universidad Estatal a Distancia. (1978). Junta Universitaria, Sesión 115 (inédito). Autor.

Universidad Estatal a Distancia. (2004a). Modelo Pedagógico. Autor.

Universidad Estatal a Distancia. (2004b). Procedimiento para la definición y administración de los materiales didácticos que se entregan a los estudiantes únicamente en calidad de préstamo (audiovisuales y multimedios). https://www.uned.ac.cr/academica/images/ cidreb/normas y procedimientos/docencia/procedimiento definicion_administ mat didacticos estudiantes en calidad prestamo.pdf

Universidad Estatal a Distancia. (2009). Estatuto Orgánico. Autor. https://www.uned.ac.cr/ academica/images/Normativa/Estatuto\%200rganico.pdf

Universidad Estatal a Distancia. (s. f.). Glosario de términos curriculares para la Universidad Estatal a Distancia. Autor. https://www.uned.ac.cr/academica/images/PACE/publicaciones/ FINAL24-9-13 Glosario de trminos curriculares UNED.pdf

Universidad Estatal a Distancia, Programa de apoyo curricular y evaluación de los aprendizajes. (2018). Guía para el diseño y rediseño curricular de asignaturas [en proceso de validación]. Autor. https://bit.ly/3gHS8zZ

Universidad Estatal a Distancia, Programa de Producción de Material Didáctico Escrito. (s. f.). Reglamento del sistema de producción de obras de la Universidad Estatal a Distancia [Material inédito]. Autor.

Villalobos-Rodríguez, V., Poltronieri-Mendez, S. y Garita-González, G. (2020). Proceso de producción de unidades didácticas y el marco UNESCO como referente para la elaboración de los materiales didácticos. Revista Electrónica Calidad en la Educación Superior, 11(1), 129157. http://dx.doi.org/10.22458/caes.v11i1.2942 